\title{
Three Different Cases of Iatrogenic Aortic Dissection Post Right Coronary Artery Angiogram
}

\author{
Marco Gennari ${ }^{1}$, Giorgio Mastroiacovo ${ }^{2}$, Gianluca Polvani ${ }^{1}$, and Marco Agrifoglio ${ }^{1}$ \\ ${ }^{1}$ Centro Cardiologico Monzino Istituto di Ricovero e Cura a Carattere Scientifico \\ ${ }^{2}$ Centro Cardiologico Monzino IRCCS
}

May 12, 2020

\begin{abstract}
Iatrogenic acute aortic dissection during percutaneous coronary intervention is an extremely rare but potentially life-threatening complication occurring in less than $<0,02 \%$ of the procedures. We report the cases of 3 patients with aortic dissection during percutaneous coronary intervention successfully treated with an emergent ascending aorta replacement. The main goal is the closing of the intimal tear as fast as possible damage either with a percutaneous system (i.e. a proximal stent) or with an open heart open surgery to prevent the extension of the dissection and neurological. A conservative strategy should be pursuit only in small localized sinus dissections.
\end{abstract}

\section{Introduction}

Iatrogenic acute aortic dissection (IAAD) during percutaneous coronary intervention (PCI) is an extremely rare but potentially life-threatening complication, occurring in less than $<0,02 \%$ of the procedures. Patients with a limited aortic wall involvement have been treated successfully by sealing the entry tear with a coronary stent. Dissections extending up the aorta $>40 \mathrm{~mm}$ from the coronary ostia require more often an emergent surgical intervention. [1]

We report three different cases of iatrogenic aortic dissection successfully treated with ascending aorta replacement.

\section{Case Presentation}

The first case is a 65-year-old Caucasian female with a past clinical history of non-ST elevation myocardial infarction (NSTEMI), who was referred to our Center for a new onset of inferior ST-elevation myocardial infarction (STEMI). She was promptly undergone percutaneous transluminal coronary angioplasty (PTCA) with implantation of four drug eluting stents (DES).

During the procedure a dissection of the right coronary artery (RCA) with the subsequent formation of an aortic wall hematoma was detected; it promptly extended in a retrograde fashion from the right sinus of Valsalva up to the ascending aorta (Figure 1 ). An attempt to occlude the intimal entry orifice was made by delivering a right ostial stent but unfortunately the dissection progressed towards the aortic arch so an emergent surgery was planned. We performed with a femoro-femoral institution of cardio pulmonary bypass (CBP) an ascending aorta replacement with a straight Dacron tube \#28 and a single coronary artery bypass $(\mathrm{CABG})$ on the right coronary artery $(\mathrm{RCA})$ with a single tract of autologous saphenous vein.

Since the weaning from CPB was unachievable due to hemodynamic instability, an Extra Corporeal Membrane Oxygenation (ECMO) implantation was necessary. [2] 
The patient was successfully weaned from the ECMO after 4 days in the Intensive Care Unit (ICU). The post-operative course was favorable and the patient was later transferred to a rehabilitation Center.

The second case is a 66-year-old male with a history of STEMI and out of hospital cardiac arrest treated with PTCA + DES on left coronary artery (LCA), complicated by the dissection of LAD itself treated with a stent re-apposition. During the following years he was subjected to multiple PTCA and DES procedures for intra-stent restenosis and ongoing severe lesions on the right coronary artery.

During the last procedure of stent deployment the patient developed a dissection of the proximal part of the RCA that rapidly extended upward until ascending aorta (Figure 2 ). An emergent computed tomography (CT) scan showed the progression of the dissection to the innominate and the left common carotid artery. He finally underwent emergency ascending aorta replacement surgery with a \#24 Dacron tube prosthesis. The postoperative course was characterized by a minor stroke on the right parietal territory with no clinical remarks at the discharge on $7^{\text {th }}$ postoperative day.

The last case is a 78-year-old male patient with a history of double emergent CABG for severe instable main trunk-related chest pain. During the transcatheter complementary revascularization occurred nearly 4 months after the surgery an intimal flap of the inner aortic wall near the right coronary ostium was detected after the injection of contrast medium. The CT scan showed an aortic dissection from the right coronary ostium to the proximal saphenous vein graft anastomosis. The patient underwent an emergency redo surgery (Figure 3 ) with replacement of ascending aorta with a \#28 straight Dacron prosthesis via a femoro-femoral cannulation; the saphenous vein graft was finally anastomosed to the marginal branch of the circumflex artery. The patient required the intra-aortic balloon pump (IABP) to be weaned from the CBP and was finally discharged to a rehabilitation center on $11^{\text {th }}$ postoperative day.

\section{Discussion}

One of the most fearsome complication that can occur during percutaneous coronary intervention is an iatrogenic acute aortic dissection that could extend retrogradely into the ascending aorta. A diseased vessel wall with multiple calcification and atherosclerotic plaques seems to be the most important predisposing factor. In most cases coronary dissection is easily diagnosed during the coronary angiography, which usually reveals a true and a false lumen, separated by a radiolucent intimal flap and a dye staining persistently localized. [3]

The process underlying IAAD is not yet completely clear; in fact there are different mechanisms involved. Firstly, the dissection may be caused by the high-pressure injection of contrast medium on a pre-existing dissection breach. Secondly shearing forces during systole and diastole could explain the propagation of the dissection in a retrograde sense. Finally, the entry breach could also be created by direct trauma of the angiographic catheters and increased by forced injection of contrast medium. [4]

The type of treatment is different depending on the type and extension of the dissection. For IAAD that remains localized at the level of the Valsalva sinus during the procedure and that extend in a retrograde form it is preferable to maintain a conservative attitude, as most tend to spontaneously regress with the collaboration of the antegrade aortic blood flow. [5]

If the dissection extends less than $40 \mathrm{~mm}$ from the coronary ostia into the ascending aorta and progresses in an antegrade fashion then it is preferable to intervene by stenting the affected coronary artery so as to close the breach and prevent the dissection from spreading. [6] The third type of strategy consists in an emergency ascending aorta replacement and is recommended if the dissection extends more than $40 \mathrm{~mm}$ from the coronary artery ostium, if the patient is hemodynamically unstable, presents with severe aortic insufficiency, has hemopericardium or if the guidewire fails to cross the occluded lesion. Coronary stenting can be useful in these cases as a "bridge to the surgery" and can avoid or reduce the progression of the dissection.

In conclusion, the goal in the treatment of IAAD should be closing the intimal tear as quickly as possible in order to prevent the progression of dissection and to avoid damage to neurological system and other end- 
organs. A percutaneous attempt is always recommended if suitable, but if it does not achieve a satisfactory result a prompt ascending aortic replacement is mandatory.

\section{References}

1. Dunning DW, Kahn JK, Hawkins ET, O'Neill WW.J.D Iatrogenic coronary artery dissections extending into and involving the aortic root. Catheter Cardiovasc Interv. 2000 Dec;51 (4):387-93.

2. Gennari M, Polvani G, Agrifoglio M. Favorable outcome of mechanical support for iatrogenic aortic dissection. Asian Cardiovasc Thorac Ann. 2019 Jan;27(1):55-57.

3. Iskandrian AS, Bemis CE, Kimbiris D, Mintz GS, Hakki AH. Primary coronary artery dissection. Chest $1985 ; 87: 227-228$.

4. Yip Hon-Kan Wu Chiung Jen Yeh Kuo Ho Hang Chi Ling Fang Chi Yuan Hsieh Kelvin Yuan Kai Fu Morgan Unusual Complication of Retrograde Dissection to the Coronary Sinus of Valsalva During Percutaneous Revascularization: A Single-Center Experience and Literature Review Chest. 2001 Feb;119 (2):493-501.

5. N Perez Castellano, MA Garcia-Fernandez, EJ Garcia, et al. Dissection of the aortic sinus of Valsalva complicating coronary catheterization: cause, mechanism, evolution, and management Cathet Cardiovasc Diagn, 43 (1998), pp. 273-279.

6. Iván J. Núñez-Gil. Incidence, Management, and Immediate- and Long-Term Outcomes After Iatrogenic Aortic Dissection During Diagnostic or Interventional Coronary Procedures Circulation. 2015 Jun 16;131 (24):2114-9.

\section{Figures}

Figure 1. Coronary angiogram showing multiple stenosis on the right coronary artery and the evidence of aortic wall dissection after stenting (A \& B, arrow). Axial thoracic computed tomography view (CT) showing the type A aortic dissection progressing to the descending aorta $(\mathbf{C} \& \mathbf{D}$, arrows $)$

Figure 2. Coronary angiogram with rapid onset and extesion of ascending aorta dissection (A \& B, arrow ) confirmed at an urgent CT scan (C \& D, arrows ).

Figure 3. Intraoperative picture. Note the extensive intimal flap within the ascending aorta just above the right coronary ostium towards the non-coronary sinus (arrow ). 

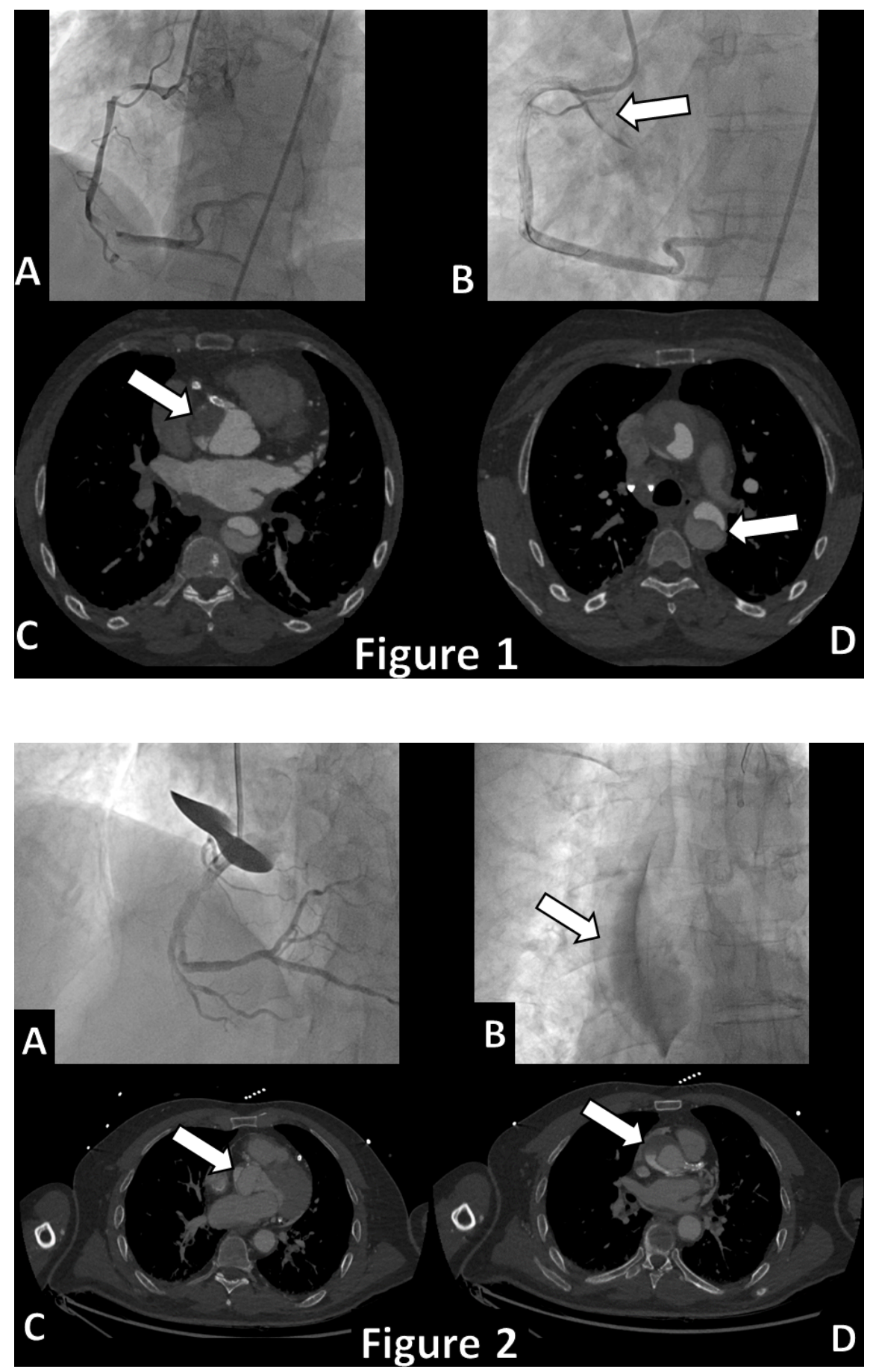


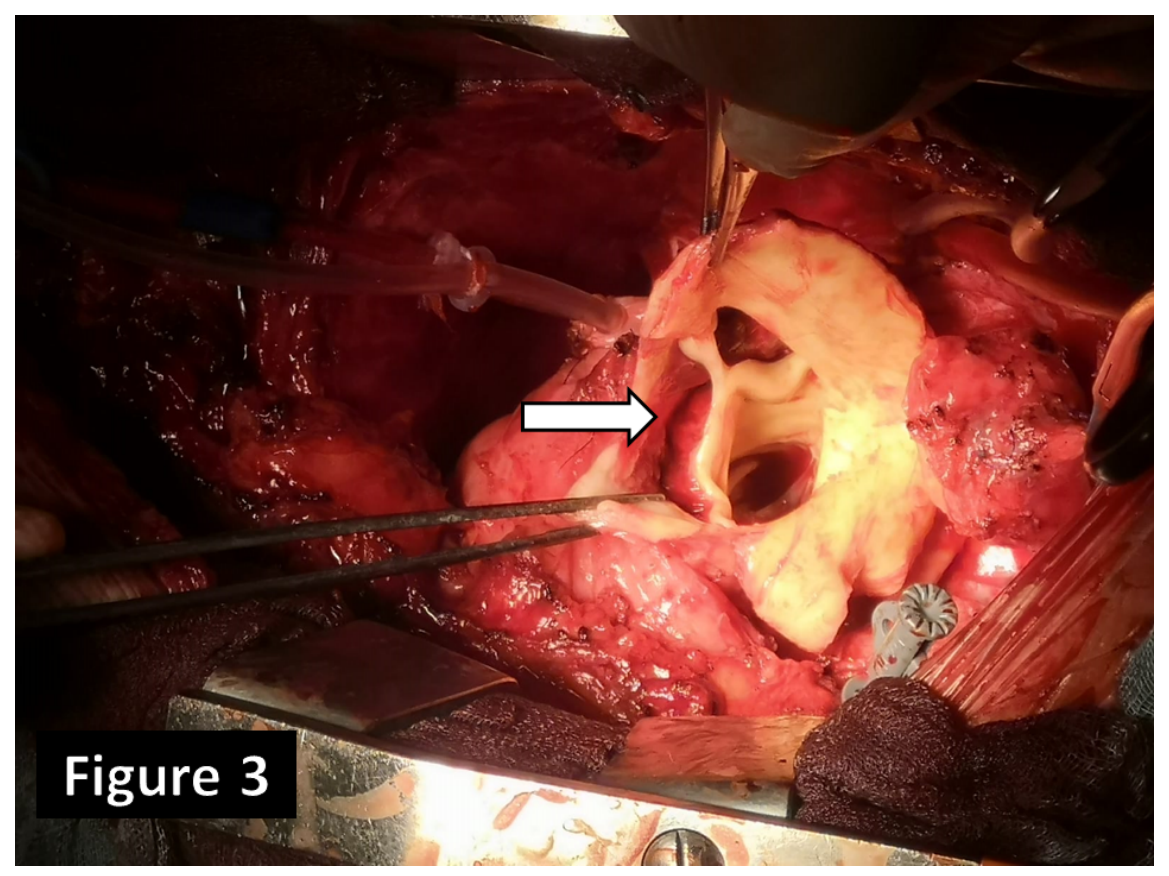

